\title{
Optical transparent infrared high absorption metamaterial absorbers
}

\author{
Jingfan Yang, Cuilian Xu, Shaobo Qu*, Hua Ma, Jiafu Wang \\ and Yongqiang Pang \\ College of Science, Air Force Engineering University \\ $X i$ 'an 710051, P. R. China \\ *qushaobo@mail.xjtu.edu.cn
}

Received 5 November 2017; Revised 11 February 2018; Accepted 11 February 2018; Published 8 March 2018

\begin{abstract}
In this work, an optical transparent infrared high absorption metamaterial absorber is proposed based on indium tin oxide (ITO) substrate. Due to the fact that ITO holds high reflectivity property in infrared region while transparent in optical region, ITO can be used in the application of Surface Plasmon Polaritons. In this design, three kinds of infrared metamaterial absorbers were proposed. All of them can achieve high absorption at $10.6 \mu \mathrm{m}$ while remaining transparent in visible region. LC equivalent circuit model was served as design foundation. The infrared absorption efficiency was numerically calculated and the mechanism analysis is given in the paper. The simulation results show that all three structures can achieve high absorption efficiency at $10.6 \mu \mathrm{m}$ under TE/TM polarization. The absorption remains high when the incident angle is less than $70^{\circ}$. Experimental results exhibit good accordance with simulation.
\end{abstract}

Keywords: ITO; infrared metamaterial absorber; visible transparent; insensitive absorption.

\section{Introduction}

The appearance of metamaterial absorbers have widened perspectives of the electromagnetic absorbers. Owing to its unique properties, metamaterial can freely modulate permeability and permittivity of material, ${ }^{1}$ which makes metamaterial absorbers achieve perfect impendence matching. Many achievements have been attained after long-term studying, and the working spectrum of metamaterial absorber has been broadened from $\mathrm{MHz}, \mathrm{GHz}, \mathrm{THz}$ to even infrared region. ${ }^{2-17}$ For example, a broadband metamaterial absorber at midinfrared using multiplexed cross-structure was proposed. ${ }^{18}$ By using metal/dielectric/metal structure with double metal ring, polarization insensitive dual-band metamaterial absorber was designed in THz region. ${ }^{19}$ Based on traditional sandwich structure, through placing multiple dielectric layers to broaden the range, broadband mid-infrared metamaterial absorbers were proposed, ultra-broadband Terahertz absorption was achieved by the uniaxial anisotropic gold nanowire arrays. ${ }^{20-24}$ However, these absorbers are usually backed by a metallic plate. The usual function of metallic backing is to provide electric boundary condition for incident plane wave, and enables creation of magnetic resonance. Besides, it is used to avoid power transmission on the other side of the absorber. The most important research in stealth material technology is aimed at compatible stealth functionality in infrared laser and visible wave bands.

So far, only highly conducting metals like Ag, Au and $\mathrm{Al}$ have been used as constituents in metamaterial absorbers. These metals have plasma frequencies at ultraviolet frequencies and highly dispersive plasma-like permittivities at infrared frequencies. Recently, doped semiconductors such as indium tin oxide (ITO) and aluminum doped zinc oxide have been used for infrared plasmonic applications. ITO thin films are good infrared reflectors while transparent at visible wavelengths, and well known for transparent electrodes in photo-voltaic applications. Due to the fact that ITO holds high reflectivity property in infrared region while transparent in optical region, ITO can be used in the application of surface plasmon polaritons. At present, the laser guidance missile usually relies on $10.6 \mu$ m laser beam to search for objects. Aimed at $10.6 \mu \mathrm{m}$ laser guidance stealth, three kinds of infrared metamaterial absorbers were proposed in our work. All of them can achieve high absorption at $10.6 \mu \mathrm{m}$ while remaining transparent in the visible region. To explain the mechanism of the infrared metamterial absorbers, LC equivalent circuit is applied, and the numerical electric field distribution is simulated. Further, all of them can remain high infrared absorption efficient under TE/TM polarization incidence with incident angle less than $70^{\circ}$.

\section{Design of Metamaterial Absorber}

The schematic of three kinds of metamaterial absorbers were shown in Fig. 1. The infrared metamaterial absorbers consist of three layers: (i) ITO pattern was on the top of the structure, (ii) the middle layer is dielectric consisting of $\mathrm{ZnS}$, (iii) the bottom layer is an ITO film which served as reflective layer. As can be seen from Fig. 1(a), when the light was thrown

This is an Open Access article published by World Scientific Publishing Company. It is distributed under the terms of the Creative Commons Attribution 4.0 (CC-BY) License. Further distribution of this work is permitted, provided the original work is properly cited. 

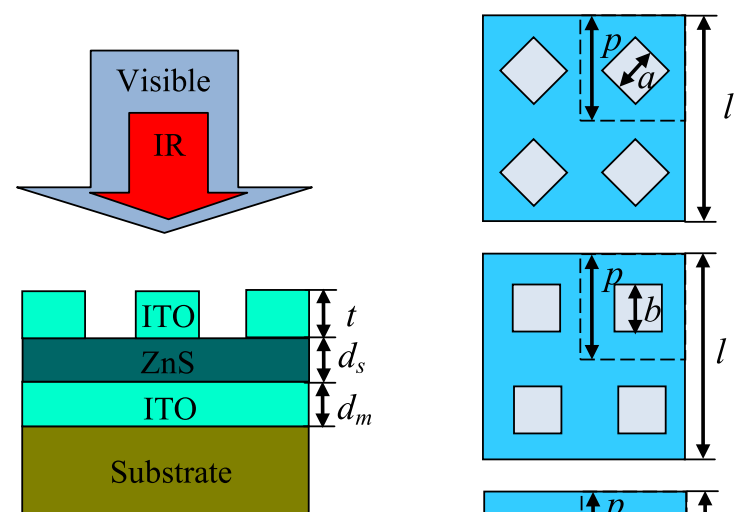

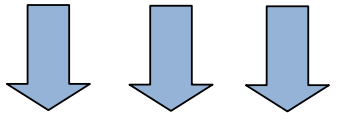

(a)
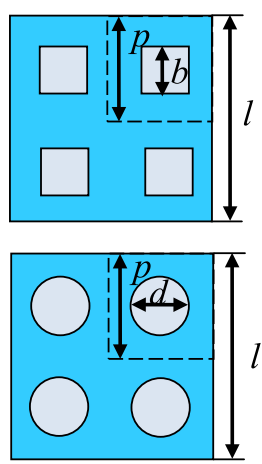

(b)
Fig. 1. Schematic graph of infrared metamaterial absorber. (a) Front view of the structure, (b) top view of three shaped structures.

upon the structures, the infrared frequency electromagnetic wave was absorbed while visible light was transmitted. Figure 1(b) shows the parameters of three structures: $l=9 \mu \mathrm{m}, p=4.5 \mu \mathrm{m}, a=2 \mu \mathrm{m}, b=2 \mu \mathrm{m}, d=2.6 \mu \mathrm{m}$, $d_{m}=200 \mathrm{~nm}, d_{s}=300 \mathrm{~nm}, t=80 \mathrm{~nm}$. The parameter of ITO film was described by Drude model with $\omega_{p}=461 \mathrm{THz}$, $\omega_{c}=28.7 \mathrm{THz}, \varepsilon_{\infty}=3.9,{ }^{25}$ the permittivity of $\mathrm{ZnS}$ was referred from Ref. 26.

In order to demonstrate the working principle of the infrared metamaterial absorber, LC equivalent circuit model was utilized. As is shown in Fig. 2, dipole LC circuit was exhibited. When the structure interacted with the incident EM waves, electric resonance and magnetic resonance occurred. Figure 2(a) represented LC circuit under electric resonance, while Fig. 2(b) represented LC circuit under magnetic resonance.
In Fig. 2, the inductance produced by induced current can be described as

$$
L=2 L_{e}=\mu\left(d_{p} / h\right) l .
$$

The capacity produced by the top pattern and bottom reflective layer can be described as

$$
C_{m}=\varepsilon_{d} S / d p
$$

where $S=h l c, c$ represented the effective factor of electric charge area.

Because the structures were in periodic array, the gap between two adjacent units can also produce capacity as the following expression:

$$
C_{e}=\pi \varepsilon_{d} h / \ln \left(g / d_{m}\right) .
$$

where $g=p-l, g$ represented the distance between two adjacent units.

According to the principle of impedance matching, the relationship between structure parameters and resonance wavelength can be described as

$$
\lambda=2 L \sqrt{\varepsilon_{d}} .
$$

where $L$ represented the size of top ITO pattern.

\section{Simulation and Experimental Validation}

CST microwave simulator was applied for the numerical calculation of three infrared metamaterial absorbers. Because all three structures held good symmetric property, the simulation boundary was set to electromagnetic boundary condition. Electric field direction was in $x$-direction, magnetic field direction was in $y$-direction. Time domain solver was utilized to simulate. $S$ parameters were numerically calculated for all three structures under TE/TM polarization incidence. The ITO film holds high reflectivity property in infrared region while transparent in optical region, ITO can be used in the application of surface plasmon polaritons, and the electromagnetic properties are similar to metals in infrared wave band.

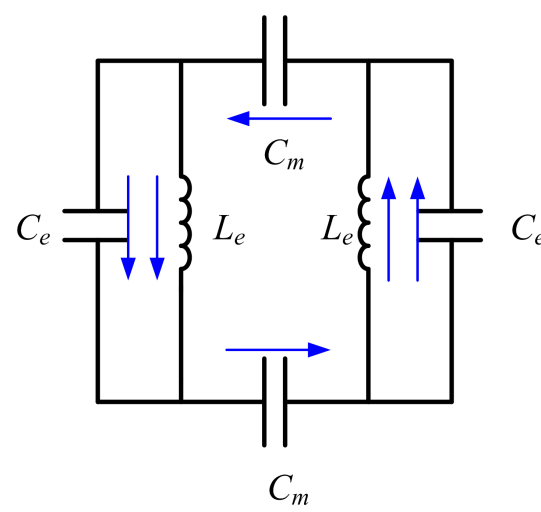

(a)

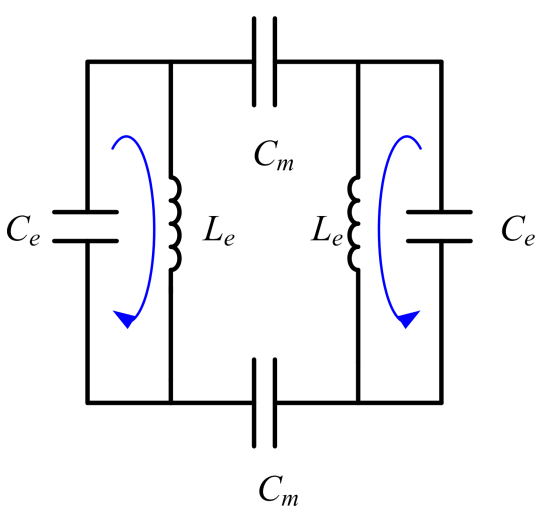

(b)

Fig. 2. Equivalent LC circuit. (a) Equivalent circuit of magnetic resonance, (b) equivalent circuit of electric resonance. 
Since the thickness of the ITO film used here is much larger than the typical skin depth, the transmission is nearly zero. Here we chose 2 inch, $10 \Omega / \mathrm{cm}$ ITO glass as substrate and the substrate has no effect on the absorber. The absorption is given by $A=1-\left|S_{11}\right|^{2}$, where $\left|S_{11}\right|$ represents the scattering parameter of reflection. The scattering parameters of the proposed structure are calculated by a commercial finite difference time domain (FDTD) method solver. The unit cell was set with periodic boundary conditions in $x-y$ plane. A normal incident plane wave along the positive $z$-direction was applied with the electric component along $x$-direction and a magnetic field along $y$-direction.

First, diamond-shaped structure was simulated under TE/TM polarization incidence. The simulation results are shown in Fig. 3. As can be seen from the figure, when the incidence was TE polarization, there existed an absorption peak at $10.56 \mu \mathrm{m}$ with the absorption efficiency of $97.3 \%$. When the incidence was TM polarization, it resonated most fiercely at $10.6 \mu \mathrm{m}$ with the absorption efficiency of $97 \%$.

Second, square-shaped structure was simulated under TE/TM polarization incidence. The simulation results are shown in Fig. 4. As can be seen from the figure, when the incidence was TE polarization, there existed an absorption peak at $10.79 \mu \mathrm{m}$ with the absorption efficiency of $96.7 \%$. When the incidence was TM polarization, it resonated most fiercely at $10.78 \mu \mathrm{m}$ with the absorption efficiency of $96.6 \%$.

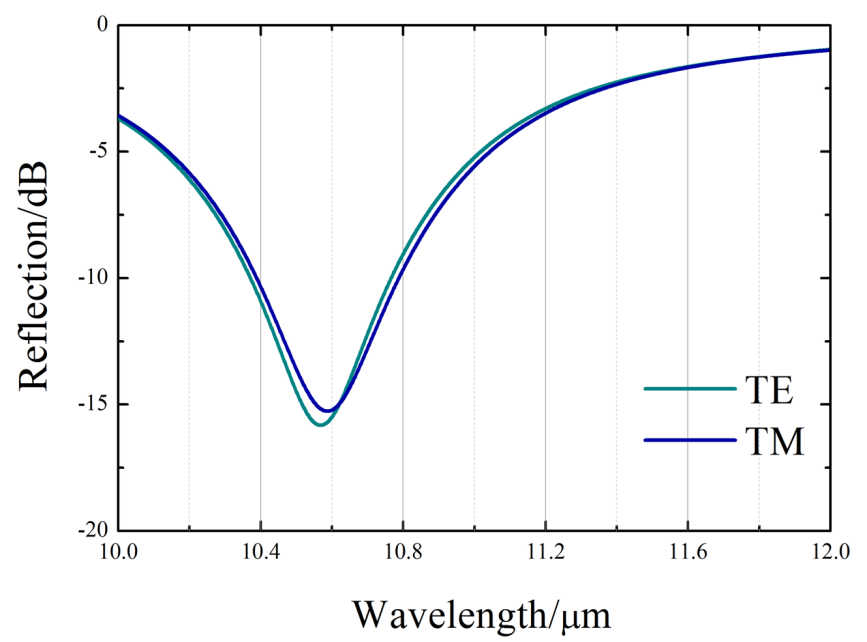

(a)

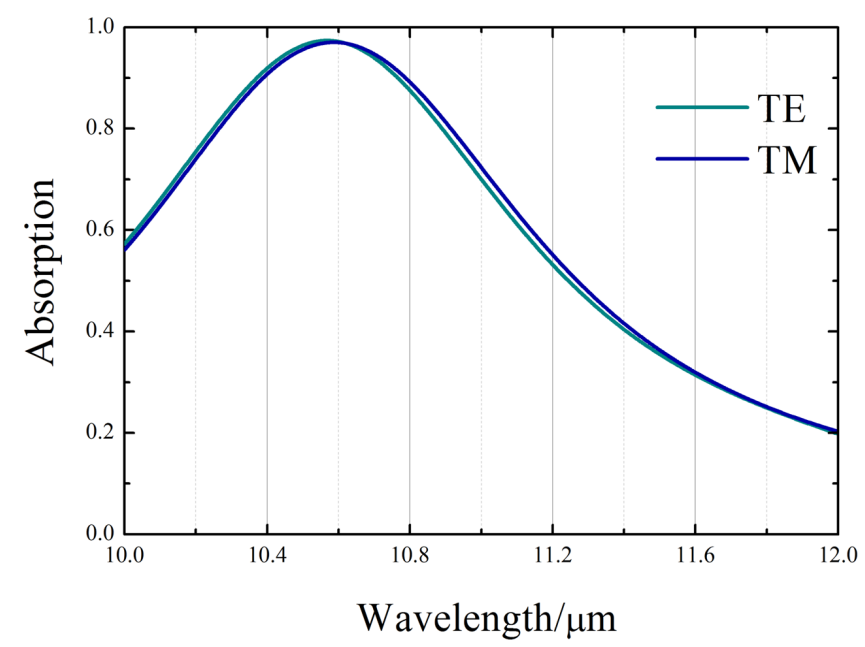

(b)

Fig. 3. Simulation results of diamond-shaped structure. (a) Reflective curve, (b) absorption curve.

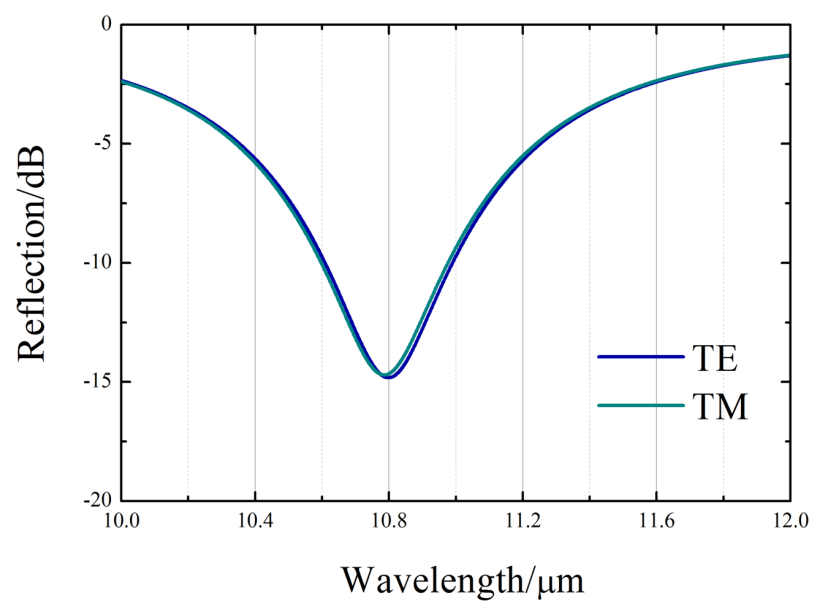

(a)

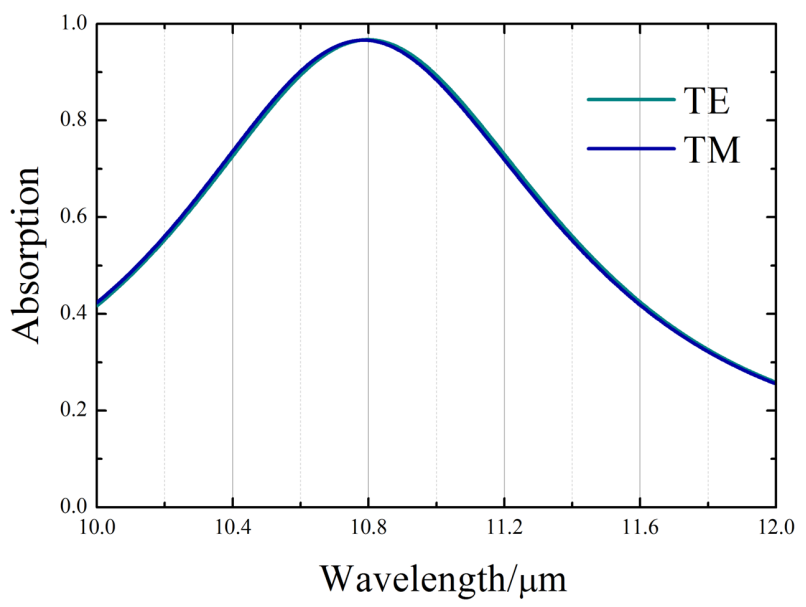

(b)

Fig. 4. Simulation results of square-shaped structure. (a) Reflective curve, (b) absorption curve. 


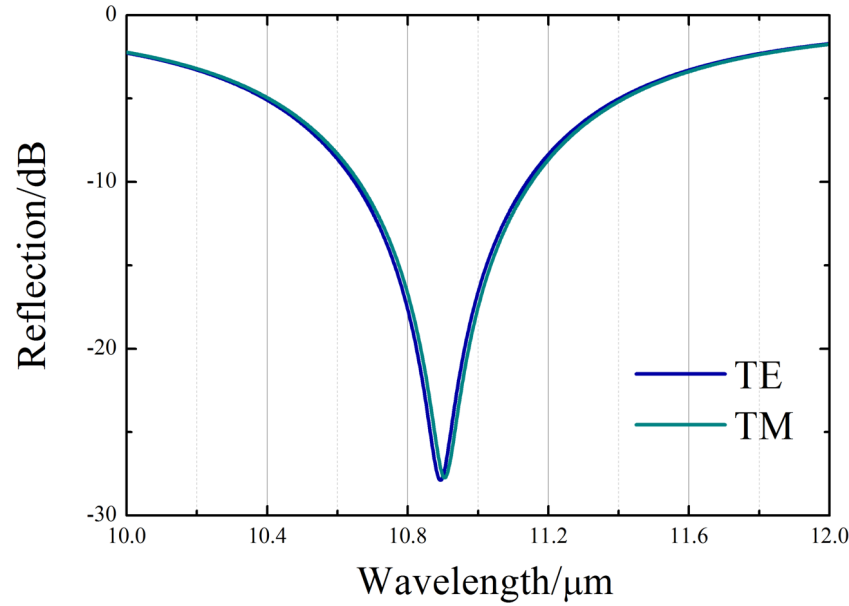

(a)

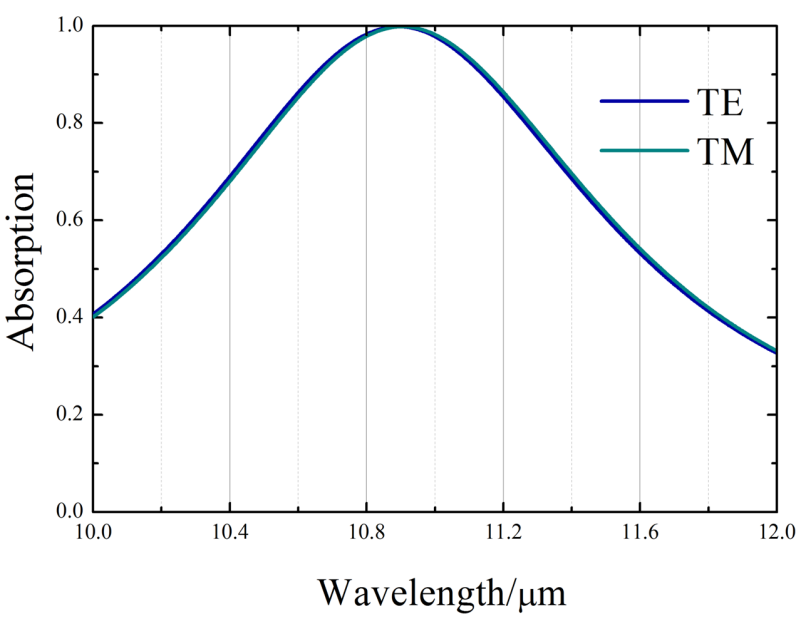

(b)

Fig. 5. Simulation results of disk-shaped structure. (a) Reflective curve, (b) absorption curve.

Third, disk-shaped structure was simulated under TE/TM polarization incidence. The simulation results are shown in Fig. 5. As can be seen from the figure, when the incidence was TE polarization, there existed an absorption peak at $10.89 \mu \mathrm{m}$ with the absorption efficiency of $99.8 \%$. When the incidence was TM polarization, it resonated most fiercely at $10.9 \mu \mathrm{m}$ with the absorption efficiency of $99.9 \%$.

From the simulated absorption curves, we can conclude that all three structures hold high absorption efficiency at around $10.6 \mu \mathrm{m}$. When the incident EM waves were vertical, there existed little difference between TE/TM polarization. All three structures were insensitive to the polarization of incidence waves, which was suitable for $10.6 \mu \mathrm{m}$ laser guidance absorption. Obviously, the disk-shaped structure absorber has the most high-absorption efficiency at around $10.6 \mu \mathrm{m}$, due to the increase of ITO duty ratio. The diskshaped structure absorber has better absorption and better polarization insensitivity feature. This can be attributed to the $360^{\circ}$ rotational symmetry of the top layer pattern.

However, taking the application reality into account, the beam of laser device is not incident perpendicular to the surface of the target. Usually, it illuminated the object at a certain angle. We further studied the absorption angle stability of the three kinds of structures. CST micro studio was

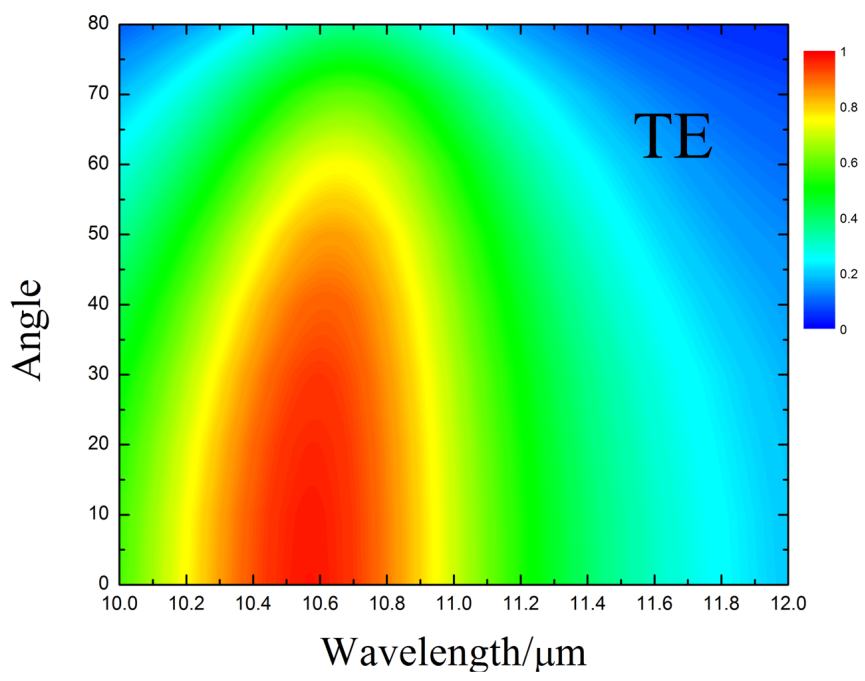

(a)

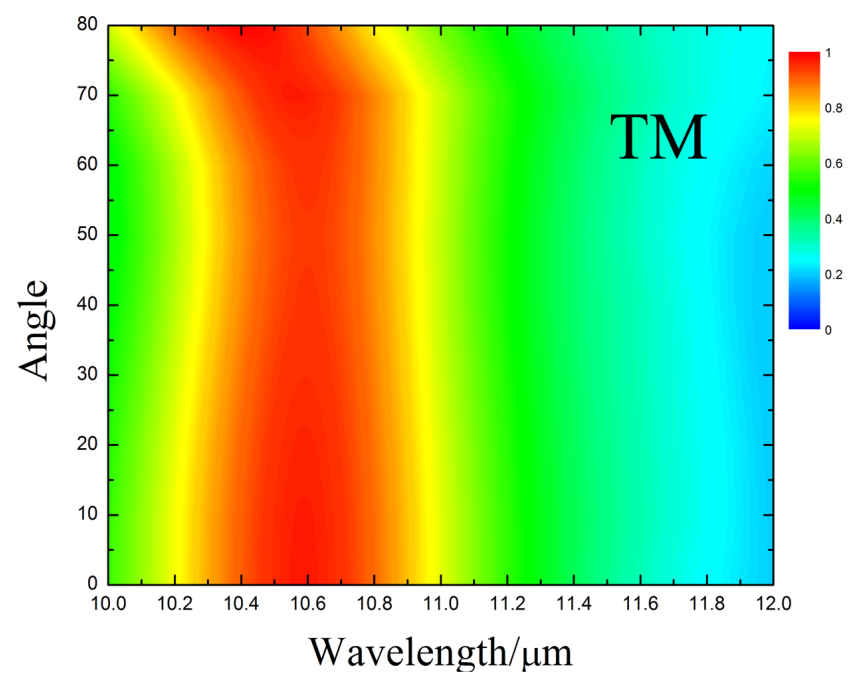

(b)

Fig. 6. Incident angle absorption contour. (a) Diamond-shaped structure incident angle absorption under TE, (b) diamond-shaped structure incident angle absorption under TM, (c) square-shaped structure incident angle absorption under TE, (d) square-shaped structure incident angle absorption under TM, (e) disk-shaped structure incident angle absorption under TE, (f) disk-shaped structure incident angle absorption under TM. 


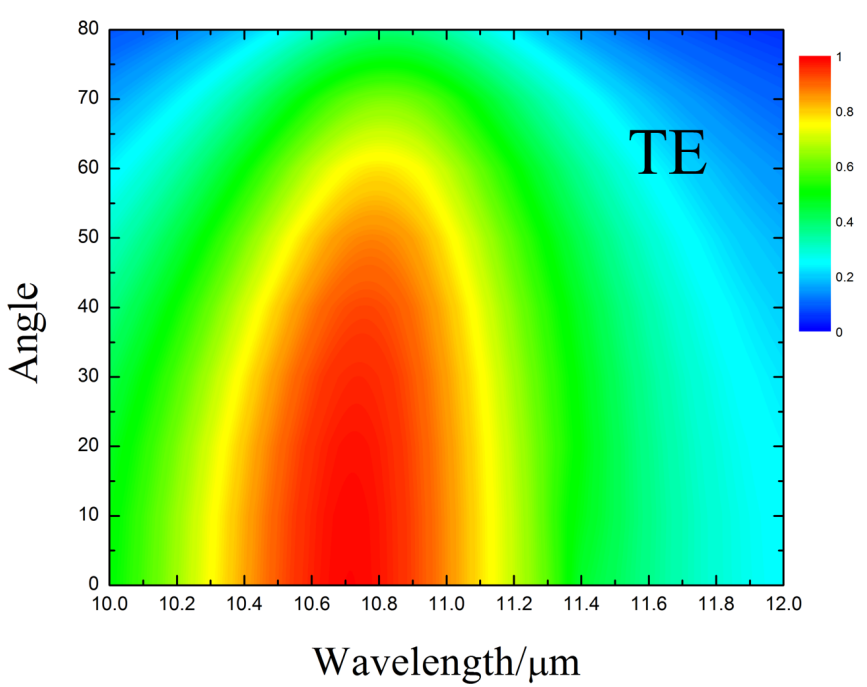

(c)

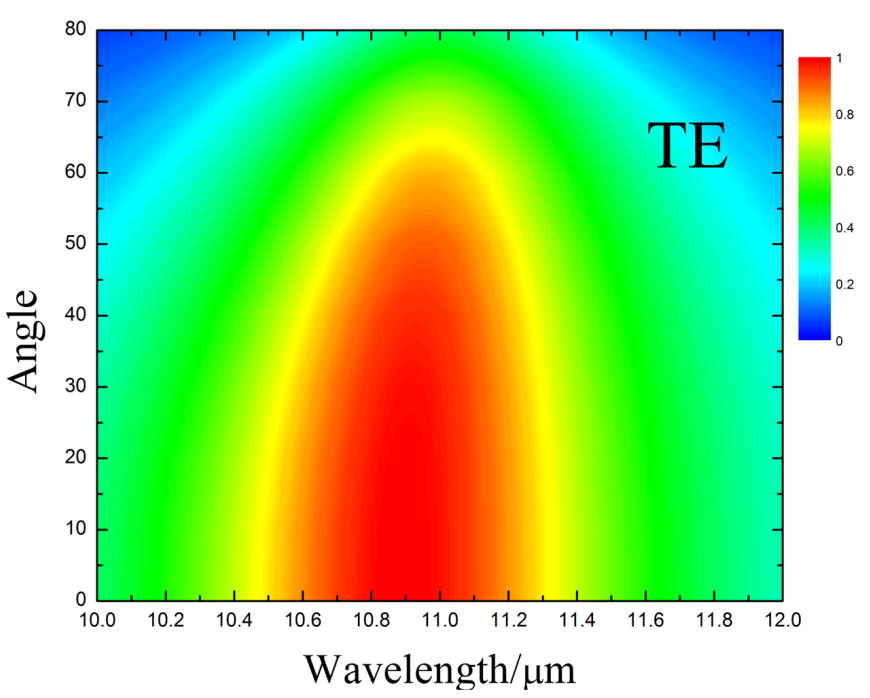

(e)

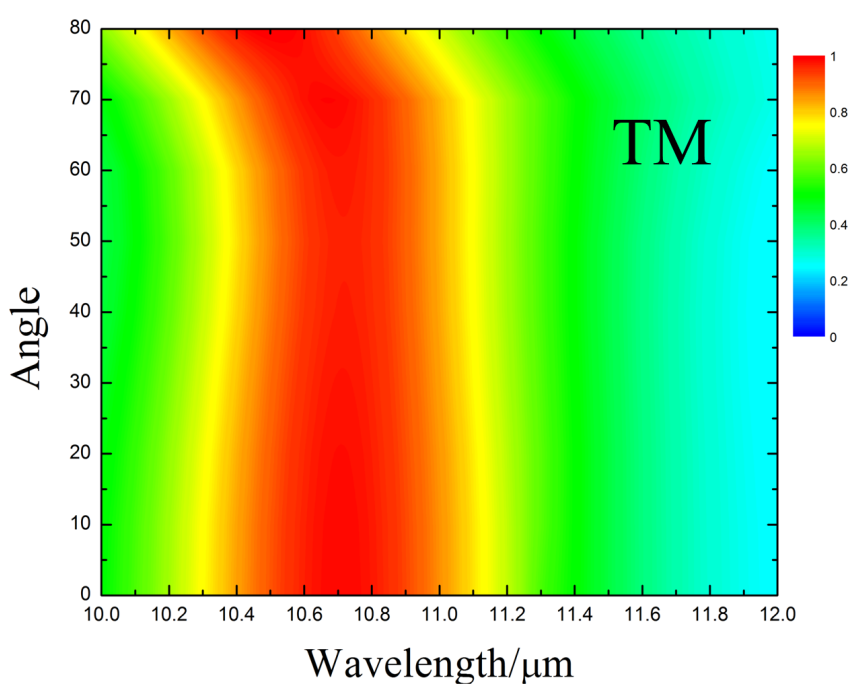

(d)

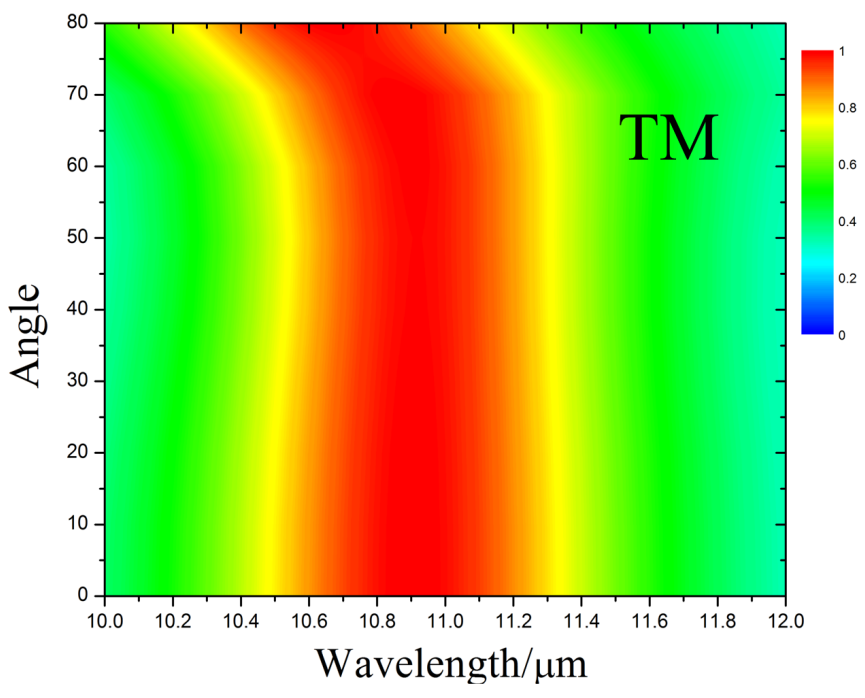

(f)

Fig. 6. (Continued)

utilized and the incident angle of simulation was set from $0^{\circ}$ to $80^{\circ}$ with $10^{\circ}$ step width. The simulation results are given in Fig. 6.

As can be seen from Fig. 6, all three structures held similar properties of incident angle under both TE and TM polarization. Supposing the incident waves were of TE polarization, the diamond-shaped structure absorption remained unchanged when the incident angle was less than $50^{\circ}$, but when the incident angle exceeded $50^{\circ}$, the absorption efficiency decreased with the incident angle increasing; the square-shaped structure absorption remained unchanged when the incident angle was less than $55^{\circ}$; as long as the incident angle exceeded $55^{\circ}$, the absorption efficiency decreased with the incident angle increasing; the disk-shaped structure absorption remained unchanged when the incident angle was less than $60^{\circ}$; once the incident angle exceeded $60^{\circ}$, the absorption efficiency decreased with the incident angle increasing. Supposing the incident waves were of TM polarization, all three kinds of structures remained high absorption efficient with the incident angle increasing. But the absorption spectrum moved towards short wavelength as the incident angle increased.

The insensitivity towards polarization of incident waves stemmed from the symmetric shape of three structures. Under TE polarization incidence, the absorption stayed unchanged because the direction of electric field of TE waves did not 
change as the incident angle increased. Under TM polarization, the electric field direction of incident waves components in $x-y$ plane decreased, which led to the decreased size of resonance. As a result, the resonant wavelength moved towards short wavelength. In addition, the absorption still remained high to some degree.

In order to further explain the mechanism of the infrared metamaterial absorber, CST micro studio field monitor was used to track the distribution of surface current and the electric field in Fig. 7. From the electromagnetic field distribution figure, we can see that the absorption property was inspired by the electric resonance and magnetic resonance caused by the three-layer structure. Thereinto, the resonate electric field of three structures were produced by the top ITO pattern and bottom ITO film. The strong resonances confined the electric field into $\mathrm{ZnS}$ middle dielectric layer. From the magnetic field distribution, there existed a strong magnetic loop in each shape of structure. The strong magnetic loop was produced by the anti-parallel currents. Thus, the electric resonance and magnetic resonance formed a strong boundary, which confined the incident wave into the three-layer structure, and the energy of waves were consumed later.

To validate our design, electron beam evaporation and UV lithography were applied to fabricate our sample. We chose 2 inch, $10 \Omega / \mathrm{cm}$ ITO glass as substrate. $300 \mathrm{~nm} \mathrm{ZnS}$ was obtained using beam evaporation under $\mathrm{Ar}=20 \mathrm{sccm}$, angle $=18^{\circ}$, sputtering pressure $=0.49 \mathrm{pa}$, and $80 \mathrm{~nm}$ ITO film under $\mathrm{Ar}=20 \mathrm{sccm}$, angle $=20^{\circ}$, sputtering pressure $=$ 0.49 pa. Top patterns were obtained through UV lithography. Figure 8 shows the measured results of three shaped structures under TE polarization. Compared with the simulation results, we found that the absorption peak exhibited a bit of movement. The experiment differences might be the inaccurate precision of the musk, which led to deviation between the sample and simulation model. Nevertheless, it can also prove that all three shaped structures can achieve high infrared absorption at $10.6 \mu \mathrm{m}$.

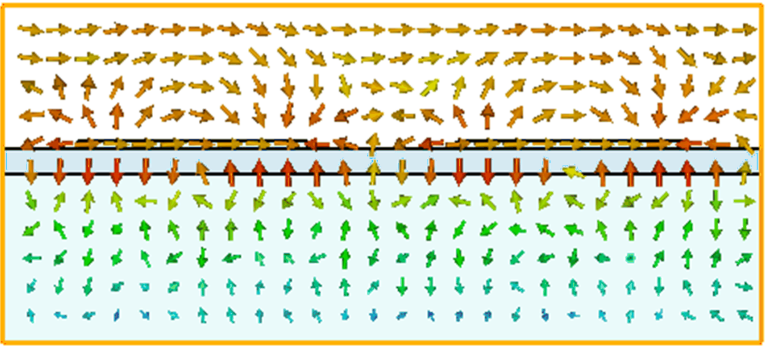

(a)

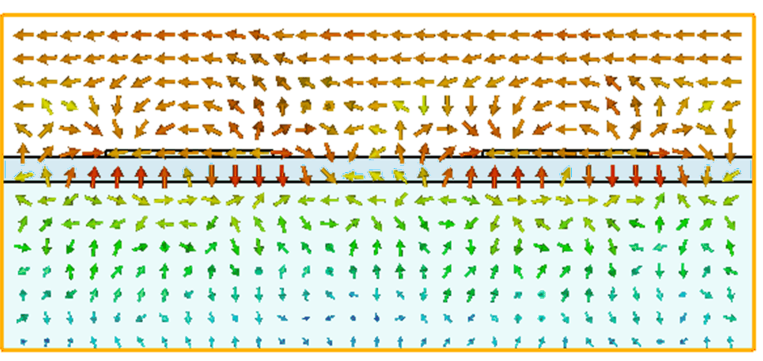

(c)

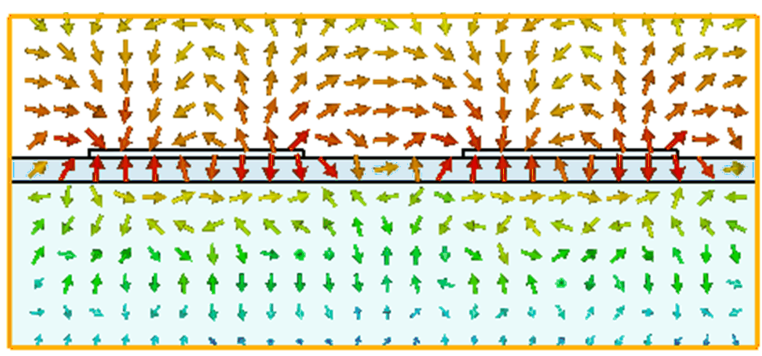

(e)
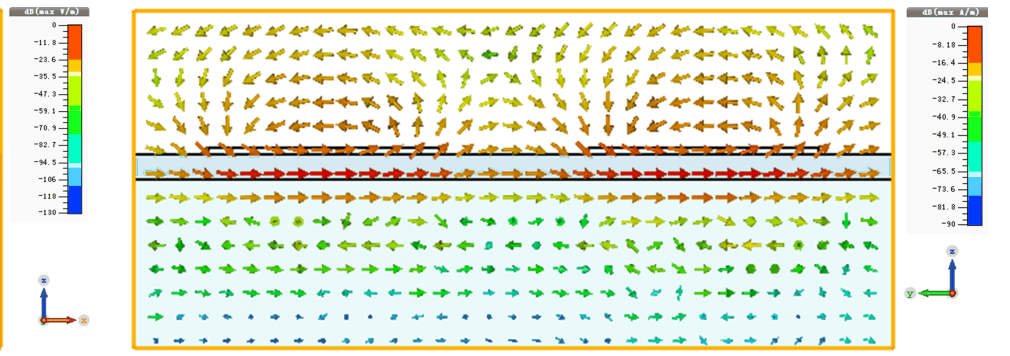

(b)
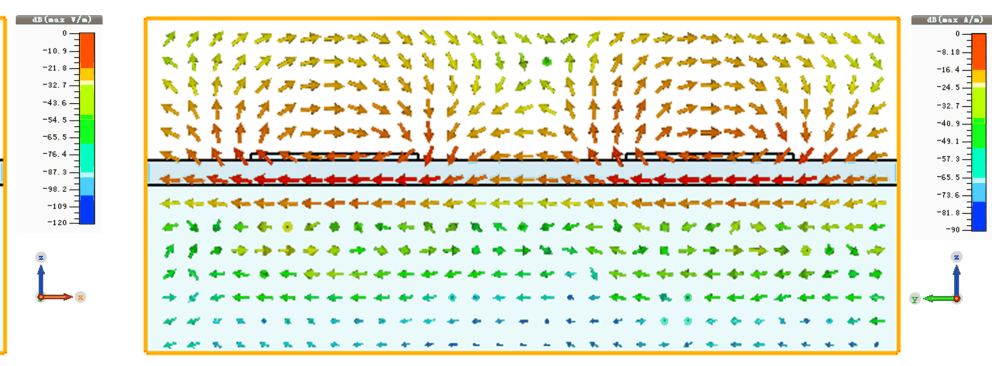

(d)
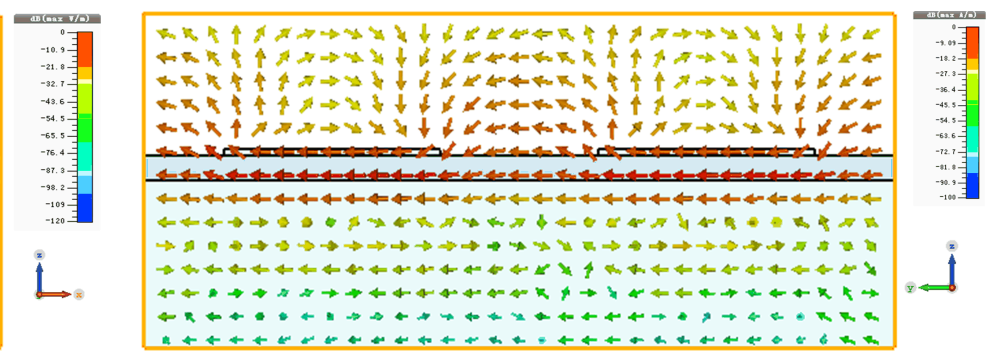

(f)

Fig. 7. Electromagnetic field distribution graph. (a) Diamond-shaped structure electric field distribution, (b) diamond-shaped structure magnetic field distribution, (c) square-shaped structure electric field distribution, (d) square-shaped structure magnetic field distribution, (e) disk-shaped structure electric field distribution, (f) disk-shaped structure magnetic field distribution. 


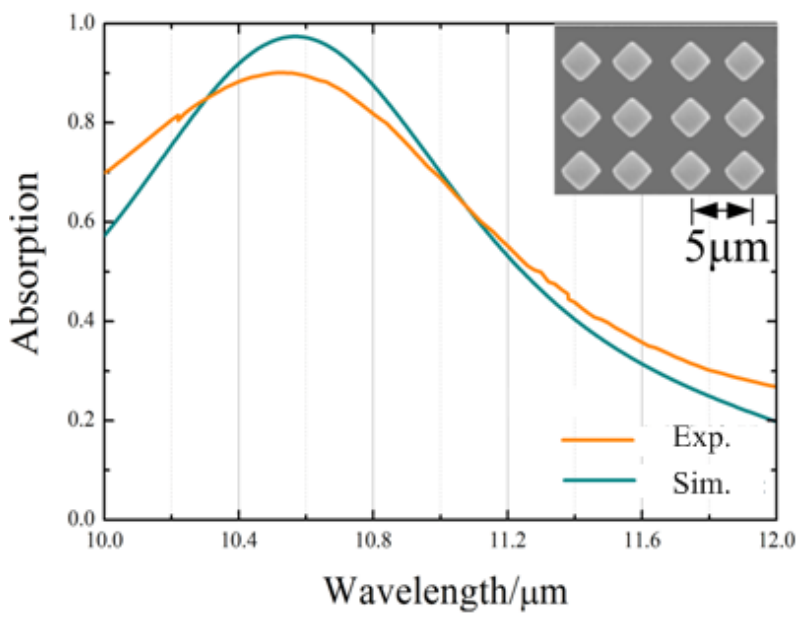

(a)

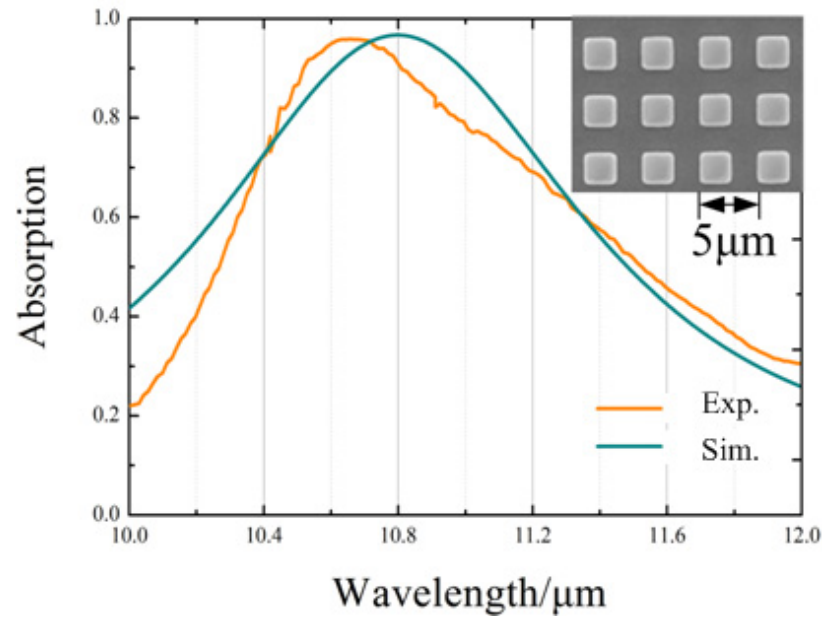

(b)

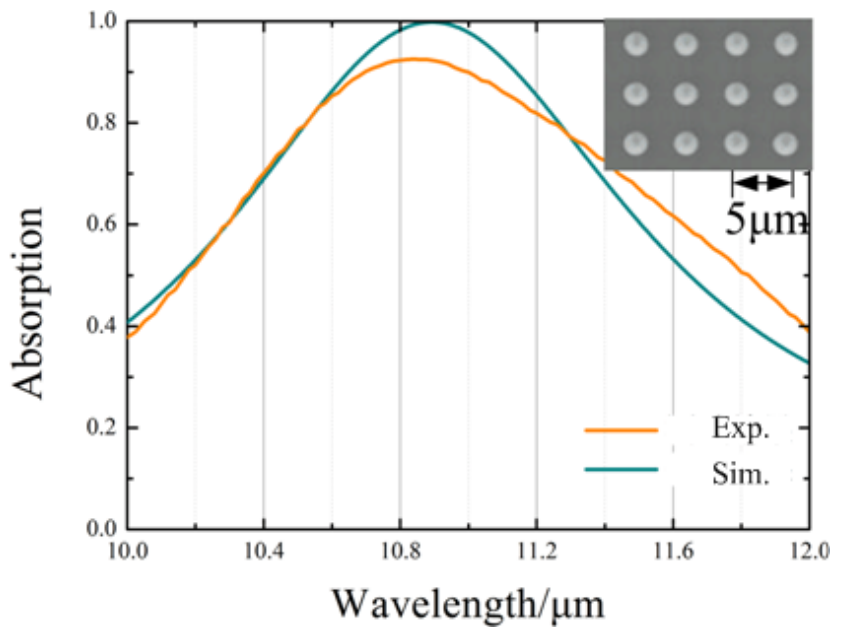

(c)

Fig. 8. The experimental results: (a) absorption curve of diamond-shaped structure, (b) absorption curve of square-shaped structure, (c) absorption curve of disk-shaped structure.

\section{Conclusion}

In this paper, three kinds of optical transparent infrared metamaterial absorbers were proposed. Those metamaterial absorbers can achieve high infrared absorption at $10.6 \mu \mathrm{m}$ both under TE and TM polarization incidence. The simulation results suggest that those infrared metamaterial absorbers can retain greater than $96 \%$ absorption over $60^{\circ}$ incident angle operating at $10.6 \mu \mathrm{m}$. The experimental results show a good accordance with the simulation results. Due to the visible transparent unique property of ITO, those infrared metamaterial absorbers might have profound application in laser guidance stealth.

\section{Acknowledgments}

The authors are grateful to the support from National Science Foundation of China under Grant Nos. 61501497, 61331005 and 61671467, and the special foundation of New-star Plan of Science and technology, No. 2015KJXX-47.

\section{References}

${ }^{1}$ J. Yang et al., Dual-band tunable infrared metamaterial absorber with VO conformal resonators, Opt. Commun. 402, 518 (2017).

${ }^{2} \mathrm{~S}$. Gu et al., A broadband low-reflection metamaterial absorber, J. Appl. Phys. 108, 064913-1 (2010).

${ }^{3}$ Y. Shen et al., An extremely wideband and lightweight metamaterial absorber, J. Appl. Phys. 117, 224503 (2015).

${ }^{4} \mathrm{~S}$. Sui et al., Two-dimensional QR-coded metamaterial absorber, Appl. Phys. A 122, 1 (2016).

${ }^{5}$ Y. J. Yoo et al., Flexible and elastic metamaterial absorber for low frequency, based on small-size unit cell, Appl. Phys. Lett. 105, 041902-1 (2014).

${ }^{6} \mathrm{H}$. Tao et al., A dual band terahertz metamaterial absorber, J. Phys. D 43, 225102 (2010). 
${ }^{7}$ C. M. Bingham et al., A metamaterial absorber for the terahertz regime: Design, fabrication and characterization, Opt. Express 16, 7181 (2008).

${ }^{8} \mathrm{~W}$. Zhu et al., Optical metamaterial absorber based on leaf-shaped cells, Appl. Phys. A 102, 147 (2011).

${ }^{9}$ A. Vora et al., Exchanging ohmic losses in metamaterial absorbers with useful optical absorption for photovoltaics, Sci. Rep. 4, 4901 (2014).

${ }^{10} \mathrm{H}$. Tao et al., A metamaterial absorber for the terahertz regime: Design, fabrication and characterization, Opt. Express 16, 7181 (2008).

${ }^{11} \mathrm{~J}$. Grant et al., Polarization insensitive, broadband terahertz metamaterial absorber, Opt. Lett. 36, 3476 (2011).

${ }^{12} \mathrm{~W}$. Zhu and X. Zhao, Metamaterial absorber with dendritic cells at infrared frequencies, J. Opt. Soc. Am. B 26, 2382 (2009).

${ }^{13} \mathrm{~S}$. Chen et al., Polarization insensitive and omnidirectional broadband near perfect planar metamaterial absorber in the near infrared regime, SPIE NanoScience Engineering. International Society for Optics and Photonics (2011), pp. 1579-1584.

${ }^{14}$ I. J. H. Mccrindle et al., Multispectral materials: Hybridisation of optical plasmonic filters and a terahertz metamaterial absorber, Adv. Opt. Mater. 2, 149 (2014).

${ }^{15}$ Y. J. Kim et al., Dual broadband metamaterial absorber, Opt. Express 23, 3861 (2015).

${ }^{16} \mathrm{X}$. Shen and T. J. Cui, Photoexcited broadband redshift switch and strength modulation of terahertz metamaterial absorber, J. Opt. 14, 114012 (2012).

${ }^{17}$ F. Dincer et al., Polarization angle independent perfect metamaterial absorbers for solar cell applications in the microwave,
Infrared, and Visible Regime, Progress in Electromagnetics Research-Pier 144, 93 (2014).

${ }^{18}$ W. Ma, Y. Wen and X. Yu, Broadband metamaterial absorber at mid-infrared using multiplexed cross resonators, Opt. Express 21, 30724 (2013).

${ }^{19} \mathrm{P}$. V. Tuong et al., Polarization-insensitive and polarizationcontrolled dual-band absorption in metamaterials, Appl. Phys. Lett. 102, 41 (2013).

${ }^{20} \mathrm{~N}$. Zhang et al., Broadband absorption in mid-infrared metamaterial absorbers with multiple dielectric layers, Opt. Commun. 338, 388 (2015).

${ }^{21}$ Y. Shen et al., Ultrabroadband terahertz absorption by uniaxial anisotropic nanowire metamaterials, IEEE Photon. Technol. Lett. 27, 2284 (2015).

${ }^{22} \mathrm{D}$. W. Hu et al., Optically transparent broadband microwave absorption metamaterial by standing-up closed-ring resonators, $A d v$. Opt. Mater. 5, 1700109-1 (2017).

${ }^{23}$ W. Li et al., Refractory plasmonics with titanium nitride: Broadband metamaterial absorber, Adv. Mater. 26, 7959 (2014).

${ }^{24} \mathrm{C}$. Long et al., Broadening the absorption bandwidth of metamaterial absorbers by transverse magnetic harmonics of 210 mode, Sci. Rep. 6, 21431 (2016).

${ }^{25}$ G. Dayal and S. A. Ramakrishna, Broadband infrared metamaterial absorber with visible transparency using ITO as ground plane, Opt. Express 22, 15104-1 (2014).

${ }^{26} \mathrm{H}$. H. Li, Refractive index of $\mathrm{ZnS}, \mathrm{ZnSe}$, and $\mathrm{ZnTe}$ and its wavelength and temperature derivatives, J. Phys. Chem. Ref. Data 13, 103 (1984). 